\title{
Quantum Rayleigh Annihilation of Entangled Photons
}

\begin{abstract}
Andre Vatarescu
Fibre-Optic Transmission of Canberra, 32 Batman Street, Canberra 2612, Australia.

Email address: andre_vatarescu@yahoo.com.au

Abstract: The interpretation of published experimental results intended to prove the existence of a quantum phenomenon of non-locality involving photonic entangled states did not take into consideration the existence of the quantum Rayleigh conversion of photons in dielectric media. This phenomenon leads to the existence of high levels of correlations between two independent photonic and linearly polarized quantum states generated after the entangled photons have been absorbed through the quantum Rayleigh conversion. Both pure and mixed individual states of polarization result in expressions normally associated with entangled photonic states, providing support for the view that the physical reality of quantum non-locality is highly questionable.
\end{abstract}

Key words: Quantum Rayleigh spontaneous emission, photon polarization states, polarization correlations.

\section{Introduction}

In an opinion article [1] published at the end of 2015, the question of quantum non-locality is all but settled on the basis of three reports published earlier in the year [2-4] apparently providing evidence of strong correlations between the two photonic subsystem components of entangled states. For some particular reason though, another report [5] of high levels of correlations between classical and entangled functions of optical polarization was totally ignored. The latter reference [5] suggests that the violations of Bell inequalities "has less to do with quantum theory than previously thought, but everything to do with entanglement."

The relatively strong correlations between the detected states of polarizations of the two space-time separated photons [1] were considered to be a clear indication of an instantaneous collapse into an eigenstate of the wave function describing the two apparently entangled photons and, as a result, it was concluded that a non-local mechanism - of an yet unknown origin and nature - brings about a mutual influence between the two distant measurements. Overall, it is argued that those correlations disprove beyond any doubt the paradox pointed out by Einstein, Podolsky and Rosen (EPR), while complying with the uncertainty principle for each subsystem which would not allow simultaneous sharp values for two incompatible variables linked to the Pauli spin operators which do not commute. However, the role of the wave functions in the evaluation of the uncertainty relation is disregarded even though the derivation of the uncertainty principle [6] is initiated with a given set of wave functions.

The measured events of correlated pairs of photons are "extremely rare" [1], with typical values of "slightly more than one event-ready signal per hour" [2]. Nevertheless, the interpretation of the experimental results of [1-5] failed to take into account the role played by the quantum Rayleigh conversion of photons [7-11] in their propagation through the dielectric media of optical fibers, beam splitters, polarization rotating devices and other dielectric elements comprising the experimental setups. While the classical Rayleigh scattering induced by perturbations of the refractive index is the major loss factor in optical fibers [12], the quantum Rayleigh conversion of photons has been practically ignored although documented in early textbooks $[7,8]$. Recently, however, the quantum Rayleigh conversion of photons has been identified as the physical process underpinning the forward propagation of an optical wave through a dielectric medium [9], as well as a practical way of implementing phasesensitive amplification in the linear regime $[10,11]$. 
The two types of experimental results [2 - 4] and [5] can be linked by recalling that a large number of photons carried by an optical wavefront emerge from either spontaneous emission or stimulated emission [9-11]. In the case of only one photon propagating through a dielectric medium, the only process occurring is that of absorption of the photon by an oscillating dipole and spontaneous emission of one photon, which corresponds to the quantum Rayleigh conversion of photons (QRCP). The QRPC would bring about various time-delays causing a photon to change direction, back and forth, inside an optical fiber or change its polarization state in any dielectric device such as optical fibers, beam splitters, crystal polarizers, etc.

From a physical perspective, the correlation between the polarization measurements at the two distant stations can be easily explained by a combination of the quantum Rayleigh spontaneous emission and the molecular structures of polarization-dependent components such as polarization beam splitters, polarization filters, birefringent crystal plates, etc. As the two measurement stations have similar, if not identical, device configurations, photons will keep propagating in their respective forward directions if they are repeatedly captured by the eigenmodes of a specific component; to a certain degree this mechanism mimics a quantum Zeno effect [6] or a protective measurement preventing a quantum state from changing [13] .

This article analyses the physical process of quantum Rayleigh scattering of photons through spontaneous emission which is bound to affect the propagation of the single photons originating from the same source and forming the components of entangled states [1-4]. As outlined in Section 2, the initially entangled state of photons is destroyed in a QRCP interaction through electric dipole excitation. The correlation functions - evaluated in Section 3 - are associated with the two spontaneously and separately emitted qubits of photons and deliver the same degree of high correlations for pure states and variable outcomes for mixed states. Additionally, each term of the commutative relations between the relevant Pauli operators in the context of the individual and separated photonic state vectors will vanish leading to the possibility of simultaneous measurements and the absence of an EPR paradox. The implications of replacing the physically eliminated entangled states of photons with individual and independent qubits are discussed in Section 4 and the Appendix below, and support the view of reference [14] objecting to the existence of quantum nonlocality. Brief conclusions summarize the main physical aspects of this physically meaningful approach. The Appendix A below describes another example of individual and independent photons replacing entangled states in explaining the effect of two-photon quantum interference.

\section{Spontaneous emission and polarization rotation}

The probability of emitting a photon with momentum $\boldsymbol{k}$ and polarization $\mu$ is related to the decay rate $\gamma_{s}[1 / \mathrm{s}]$ of the excited dipole inside a dielectric medium, and evaluated as [15]:

$\gamma_{S}^{(\text {free) }}(\boldsymbol{k}, \mu, \omega)=\frac{9 \varepsilon^{5 / 2}}{(2 \varepsilon+1)^{2}} \frac{\omega^{3}}{\hbar c}\left(\frac{\boldsymbol{d} \cdot \boldsymbol{e}_{\boldsymbol{k} \mu}}{4 \pi}\right)^{2}$

with $\boldsymbol{d}$ denoting the electric dipole moment vector which is excited by an optical field of the same polarization, $\boldsymbol{e}_{\boldsymbol{k} \mu}$ being the polarization unit vector of the emitted photon, and which is perpendicular to the direction of propagation $\boldsymbol{k}$. In a dielectric material of constant $\varepsilon$ the decay rate is modified, but its angular distribution is the same as in free space.

The angular distribution of an accumulated number of spontaneously emitted photons $N_{s p}\left(\Delta z, \varphi_{e m}\right)$ over a distance $\Delta z$ is found from Eq. (1), leading to:

$$
N_{s p}\left(\Delta z, \varphi_{e m}\right)=N_{s p}(\Delta z)\left(\cos \varphi_{e m}\right)^{2}
$$


with $\varphi_{e m}$ the emission angle between the dipole $\boldsymbol{d}$ and the polarization unit vector $\boldsymbol{e}_{\boldsymbol{k} \mu}$ of the photons and $N_{s p}(\Delta z)$ is calculated as in reference [9]. Spontaneously emitted photons with $\pm \varphi_{\text {em }}$ polarization angles relative to the pump polarization $\boldsymbol{e}_{p}$, will be amplified through the optically linear parametric gain coefficient $[10,11]$ which will include a polarization dependence in the factor $\boldsymbol{e}_{p} \cdot \boldsymbol{e}_{\boldsymbol{k} \mu}$, bringing about a correlation between the state of polarization and its number of amplified photons as found in [5]. To facilitate the reader's understanding of the quantum Rayleigh conversion of photons and its amplifications of spontaneous emission, the corresponding equations of motion are reproduced in Appendix B below.

For $\boldsymbol{e}_{\boldsymbol{k} \mu} \cdot \boldsymbol{x}=\cos \varphi_{e m}$ and $\boldsymbol{x} \cdot \boldsymbol{y}=0$, a non-vanishing value along the $\boldsymbol{y}$-polarization is obtained by blocking off either $+\varphi$ or $-\varphi$ polarized photons as, for a large number of photons, the $\boldsymbol{y}$-polarized photons cancel each other out.. This corresponds to the use of a polarization filter for the polarization paradox which "rotates" photons from $\boldsymbol{x}$ to $\boldsymbol{y}$.

The generic eigenstates of polarization associated with spontaneous emission through quantum Rayleigh conversion of photons on the two-dimensional Hilbert space $\mathcal{H}$ will take the form of single and independent qubits $\left|\Psi\left(\varphi_{\mathrm{em}}\right)\right\rangle$ identified as:

$$
\left|\Psi\left(\varphi_{e m}\right)\right\rangle=\cos \varphi_{e m}|x\rangle+\sin \varphi_{e m}|y\rangle
$$

These state vectors with polarization angles $\varphi_{e m}$ in the range $-\pi / 2 \leq \varphi_{e m} \leq \pi / 2$ will describe any possible polarization perpendicular to the direction of propagation of the spontaneous emission and will be of practical interest in the next Section. Thus, incoming photons initially polarized in the $x$-direction will reappear with an angle $\theta$ - rotated polarization, thereby enabling them to pass through a $\theta$-rotated polarization analyzer.

\section{Correlation functions}

As a photon enters a birefringent crystal and interacts with electric dipoles, the photon needs to be re-emitted into a polarization eigenstate so it can propagate in the same forward direction to reach the intended photodetector. If each of the individual photons of the initial pair is reemitted into their original state of polarization and reaches its respective detector within the designated time interval for a coincidence count to be registered, then this physical process can be mistaken for the physically impossible case of the entangled photons having survived their propagation through the dielectric media without interacting with electric dipoles. Nevertheless, as photons acquire a phase shift as a results of their propagation, the probability of no dipole-photon interactions taking place even for a short distance of millimetres, is nil.

\subsection{Pure states of polarization}

Although the conventional definition of the correlation function - see [16] (Eq.13) - involves the same state of polarization reaching the two separate detectors, in the case of quantum Rayleigh spontaneous emission additional correlations can be defined between different states of polarization - possibly boosting the detection counts - for two different angles $\varphi_{1}$ and $\varphi_{2}$, relative to the $x$ - axis of reference. Correlation functions $E_{c}$ for a quantum behaviour are defined [16] as the expectation value of the tensor product of two measurement operators for a set of initial state vectors

$$
E_{c}=\left\langle\Psi\left(\varphi_{1}\right)\left|\hat{p}\left(\theta_{1}\right) \otimes \hat{p}\left(\theta_{2}\right)\right| \Psi\left(\varphi_{2}\right)\right\rangle=\left\langle\Phi_{1}|\otimes| \Phi_{2}\right\rangle
$$


where the initial state vector $\left|\Psi\left(\varphi_{j}\right)\right\rangle$ of Eq. (3) is modified by the measurement operators $\hat{p}\left(\theta_{j}\right)$, $j=1,2$ so that, $\left|\Phi_{j}\right\rangle=\hat{p}\left(\theta_{j}\right)\left|\Psi\left(\varphi_{j}\right)\right\rangle$. This notation is equivalent to the definition of the correlation functions for a quantum behaviour [16] (Eq.13) of measurements performed at two different locations for pure states and $\varphi_{1}=\varphi_{2}$. The polarization eigenstates of the measured photons, rotated by an angle $\theta_{j}$ from the reference or generic states $|x\rangle$ and $|y\rangle$ are denoted as $\left|x\left(\theta_{j}\right)\right\rangle=\cos \theta_{j}|x\rangle+\sin \theta_{j}|y\rangle$ and $\left.\left|y\left(\theta_{j}\right)\right\rangle=-\sin \theta_{j} x\right\rangle+\cos \theta_{j}|y\rangle$ and the quantum operator measuring polarization properties of the photons is the projector

$$
\begin{aligned}
\hat{p}\left(\theta_{j}\right) & =\left|x\left(\theta_{j}\right)\right\rangle\left\langle x\left(\theta_{j}\right)|-| y\left(\theta_{j}\right)\right\rangle\left\langle y\left(\theta_{j}\right)\right| \\
& =\sin \left(2 \theta_{j}\right) \hat{\sigma}_{1}+\cos \left(2 \theta_{j}\right) \hat{\sigma}_{3}
\end{aligned}
$$

where $\hat{\sigma}_{1}=|x\rangle\langle y|+| y\rangle\langle x|$ is the real part of the two-dimensional Pauli transition operator, flipping the photon between the two generic eigenstates, and $\hat{\sigma}_{3}=|x\rangle\langle x|-| y\rangle\langle y|$ corresponds to the Pauli projection operator for the difference between the generic eigenstates. Using the identities of the operators: $\hat{\sigma}_{1}|x\rangle=|y\rangle ; \hat{\sigma}_{1}|y\rangle=|x\rangle ; \hat{\sigma}_{3}|x\rangle=|x\rangle ; \quad \hat{\sigma}_{3}|y\rangle=-|y\rangle$, $\hat{\sigma}_{1} \hat{\sigma}_{3}=-\hat{\sigma}_{3} \hat{\sigma}_{1}$ and $\hat{\sigma}_{1} \hat{\sigma}_{1}=\hat{\sigma}_{3} \hat{\sigma}_{3}=\hat{I}=|x\rangle\langle x|+| y\rangle\langle y|$ we obtain from Eq. (5) for the correlation operator:

$$
\hat{p}\left(\theta_{1}\right) \otimes \hat{p}\left(\theta_{2}\right)=\cos 2\left(\theta_{1}-\theta_{2}\right) \hat{I}+\sin 2\left(\theta_{1}-\theta_{2}\right) \hat{\sigma}_{1} \hat{\sigma}_{3}
$$

By inserting Eq. (6), along with the equalities $\left\langle\Psi\left(\varphi_{1}\right) \mid \Psi\left(\varphi_{2}\right)\right\rangle=\cos \left(\varphi_{1}-\varphi_{2}\right)$ and $\left.\left\langle\Psi\left(\varphi_{1}\right)\left|\hat{\sigma}_{1} \hat{\sigma}_{3}\right| \Psi\left(\varphi_{2}\right)\right\rangle=\left\langle\Psi\left(\varphi_{1}\right) \mid \Psi\left(\varphi_{2}+\pi / 2\right)\right\rangle=\cos \left(\varphi_{1}-\varphi_{2}-\pi / 2\right)\right)=\sin \left(\varphi_{1}-\varphi_{2}\right)$ into Eq. (4), we evaluate the correlation function $E_{c}$ to be:

$E_{c}=\cos 2\left(\theta_{1}-\theta_{2}\right) \cos \left(\varphi_{1}-\varphi_{2}\right)+\sin 2\left(\theta_{1}-\theta_{2}\right) \sin \left(\varphi_{1}-\varphi_{2}\right)$

$E_{c}=\cos \left[2\left(\theta_{1}-\theta_{2}\right)-\left(\varphi_{1}-\varphi_{2}\right)\right]$

For $\varphi_{1}=\varphi_{2}$, this expression of the correlation function for single and independent qubits of the same state of polarization reaching both detectors, is identical to the expression for photonic entangled Bell states [17] (Ch.19), reaching the two detectors. Equally, Eq. (7) evaluates the correlation for the orthogonal detections, i.e., $\theta_{1}-\theta_{2}=\pi / 2$, of two different states of photon polarizations. With adjustable settings of the detecting polarization filters, i.e. $\theta_{1}$ and $\theta_{2}$, any values of the correlation functions can be obtained [2 - 4]) for corresponding values of the incoming photon polarization angles, i. e., $\varphi_{1}$ and $\varphi_{2}$.

The detection of photons having a polarization direction $\boldsymbol{e}_{\boldsymbol{k} \mu}$ which is not aligned with the polarization filter $\boldsymbol{e}_{f}$ will occur due to the probability of a dipole excitation being proportional to the scalar product $\boldsymbol{e}_{f} \cdot \boldsymbol{e}_{\boldsymbol{k} \mu}[7,8]$. For photons to propagate in the same forward direction in a uniaxial crystal they need to be recaptured after spontaneous emission by the electric dipoles which are aligned with the principal axes of the crystal.

A relation can be derived between the correlation function of the measurements $E_{c}\left(\theta_{1}, \theta_{2}\right)$ $=\cos 2\left(\theta_{1}-\theta_{2}\right)$ and the overlap probability $P\left(\theta_{1}, \theta_{2}\right)=\left|\left\langle\Psi\left(\theta_{1}\right) \mid \Psi\left(\theta_{2}\right)\right\rangle\right|^{2}=$ 
$\cos ^{2}\left(\theta_{1}-\theta_{2}\right)$ before the measurements of two independent photons having polarization angles of $\theta_{1}$ and $\theta_{2}$. As in [17] (Ch.19), this relation is $E_{c}\left(\theta_{1}, \theta_{2}\right)=2 P_{(}\left(\theta_{1}, \theta_{2}\right)-1$ indicating that entangled states of photons do not possess any particular properties regarding quantum correlations associated with detections at two remote locations.

\subsection{Mixed states of polarization}

The overall correlation for one step of spontaneous emission will be found by adding up probability-weighted correlation functions of Eq. (7) as the ensemble of polarizations states generated over a time interval corresponds to a mixed quantum state described by the density matrix elements $\rho_{m n}(\varphi)=p(\varphi)\langle m \mid \Psi(\varphi)\rangle\langle\Psi(\varphi) \mid n\rangle$, where $m, n=x, y$. A possible probability density can be identified from Eq. (2) above, that is, $p(\varphi)=(\cos \varphi)^{2} /(0.5 \pi)$, for generating the state $|\Psi(\varphi)\rangle$ over the range $\varphi \in\{-\pi / 2, \pi / 2\}$. This leads to a higher probability for the interval $\{-\pi / 4, \pi / 4\}$ than for the intervals $\{-\pi / 2,-\pi / 4\}$ and $\{\pi / 4, \pi / 2\}$.

The correlation function for the mixed state of an ensemble is evaluated similarly to Eq. (7) after using the transformation $|\Psi(\varphi)\rangle \rightarrow[p(\varphi)]^{1 / 2}|\Psi(\varphi)\rangle$ in Eq. (4) to obtain:

$$
\begin{aligned}
& E_{c}=\cos \left[2\left(\theta_{1}-\theta_{2}\right)\right]+\int d \varphi_{2} \sqrt{p\left(\varphi_{2}\right)} \times \\
& \quad \times \int_{-\pi / 2}^{\pi / 2} \sqrt{p\left(\varphi_{1}\right)} \cos \left[2\left(\theta_{1}-\theta_{2}\right)-\left(\varphi_{1}-\varphi_{2}\right)\right]\left[1-\delta\left(\varphi_{1}-\varphi_{2}\right)\right] d \varphi_{1}
\end{aligned}
$$

where the first term reproduces the result for identical and independent qubits, i.e., $\varphi_{1}=\varphi_{2}$, with $\delta$ being Dirac's delta function, and the second term depends on the polarization state distribution of the mixed state, providing the possibility of controlling the level of correlation with various distributions of polarizations.

As the expectation values of the operator products of Eq. (6) are found to vanish for identical pure states of Eq. (3), i.e., $\left\langle\Psi(\varphi)\left|\hat{\sigma}_{3} \hat{\sigma}_{1}\right| \Psi(\varphi)\right\rangle=0$ and $\left\langle\Psi(\varphi)\left|\hat{\sigma}_{l} \hat{\sigma}_{3}\right| \Psi(\varphi)\right\rangle=0$, because $\hat{\sigma}_{l} \hat{\sigma}_{3}|\Psi(\varphi)\rangle=|\Psi(\varphi+\pi / 2)\rangle$, each term of the resulting commutative relation vanishes and we obtain:

$$
\left\langle\Psi(\varphi)\left|\left[\hat{\sigma}_{1}, \hat{\sigma}_{3}\right]\right| \Psi(\varphi)\right\rangle=0
$$

The eigenstates of $\hat{\sigma}_{1}$ are superpositions of the eigenvectors of $\hat{\sigma}_{3}$ on the two-dimensional Hilbert space $\mathcal{H}$ and simultaneous measurements of well-defined values are possible as their product operator $\hat{\sigma}_{1} \hat{\sigma}_{3}$ flips the eigenstates $|\Psi(\varphi)\rangle$ and $|\Psi(\varphi+\pi / 2)\rangle$ onto each other. Thus, the output value is indicative of the input one, and each term of the commutator vanishes for the wave functions $|\Psi(\varphi)\rangle$ of Eq .(3). Consequently, the simultaneous measurement of the two operators in the context of the single and independent qubit wave functions is capable of identifying the incoming state as well as the measured one.

\section{Physical aspects of simultaneous measurements of independent photons}

Since the same correlation functions are derived for independent and single qubits generated through quantum Rayleigh conversion of photons - from initially entangled polarized photons - as for the initially entangled photons, it follows that the violations of any type of relevant Bell inequalities will also take place in the same way. Yet, the correlations result from similar, if 
not identical, distributions of polarization states as opposed to what is conceptually believed to be a non-local quantum effect which has an unspecified nature but is being pursued because of vested interests.

Once the same correlation functions are derived using only states of polarizations emitted spontaneously by the quantum Rayleigh conversion of photons, no other physical processes is required to explain the experimental results.

Let us now consider a few characteristics associated with local realism [6] of quantum measurements in the context of quantum Rayleigh conversion of photons:

Locality of measurements is supported by the use of single and independent photonic qubits emitted separately to explain the experimental results of apparently enhanced correlations of outcomes.

Randomness of experimental parameters stems from the quantum Rayleigh spontaneous emission that generates the projection from the polarization state $|x\rangle$ of the input photons to the rotated polarization state $|\Psi(\varphi)\rangle=\cos \varphi|x\rangle+\sin \varphi|y\rangle$.

Realism of values carried by the detected photons is indicated by the physical effect of the measuring operators on the detected photons in quantum states $\left|\Psi\left(\varphi_{j}\right)\right\rangle$ of Eq. (3) for which the two commutator terms of the two Pauli operators of Eqs. (9) vanish independently of each other. Thus, a physically meaningful identification of wavefunctions will enable simultaneous measurements of well-defined values.

The common view [6] holds that "the measurement of one component of the entangled state collapses the total wave function into a certain value which, in turn, affects instantaneously the second measured value." Nonlocality is associated with the instantaneous collapse of the wave function. The "remarkable" correlation is revealed by a comparison of the two lists of measured data compiled at the two detection points as ethereal influences are said to be associated with the collapse of the wave function upon measurement. Yet, the experimental results can be explained without entangled states of photons which are destroyed by propagating through a dielectric medium and replaced by independent qubits of photon polarization.

The presentation of [17] (Ch.19) describes the Einstein, Podolsky and Rosen (EPR) view suggesting that there is no such thing as an uncaused random event, and the characteristic randomness of the quantum world originates at the very beginning of each macroscopic event. By contrast, the conventional view [1] would have a quantum description in which the state vector evolves in a perfectly deterministic way from its initial value, and randomness enters only at the time of measurements. The quantum Rayleigh spontaneous emission is, in fact, a random process at the generating stage followed by evolution described by the Schrödinger equation, thereby supporting the EPR view.

It is emphasized in [5] that "Bell violation has less to do with quantum theory than previously thought, but everything to do with entanglement." Actually, there is no need for entangled states to measure strong correlations of polarization between spontaneously emitted photons detected far apart from each other or non-locally.

It is claimed in [16] that "... the violation of Bell inequalities can be seen as a detector of entanglement that is robust to any experimental imperfection: as long as a violation is observed, we have the guarantee, independently of any implementation details, that the two systems are entangled." Yet, this is not the case with single and independent qubits which can reproduce the same results.

For the entangled state of two polarized photons shown in the inset of [1] (Fig. 1), quantum mechanics predicts that the polarization measurements performed at the two distant stations will be strongly correlated [1]. But the same prediction also applies to two independent, single qubits which are generated through quantum Rayleigh spontaneous emission from initially 
identical photons propagating in different directions through dielectric media such as optical fibers.

Additionally, reference [18] “...rules out outcome-dependent causal models without additional assumptions in any scenario with more than two settings. A direct causal influence from one outcome to the other can therefore not explain quantum correlations."

The analysis presented in this Letter is based on physically meaningful interactions of quantum Rayleigh conversion of photons and supports reference [14] in its statement that "There is no mystery. There is no quantum nonlocality". It is the physical process that gives rise to a wave function. The opposite approach of relying on mathematical complexities to conjure up physical processes is bound to generate "quantum mysteries".

As for the quantum key distribution between the two measuring units [19], it is determined by the local distribution of the mixed state of spontaneously emitted photons and the measurement setup of the dielectric devices involved in the polarization filtering with its eigenstates capturing the projected single qubits. However, errors will appear because of the statistical nature of the correlations between polarized photons.

The opinion article of reference [20] does not deal with the quantum effect of Rayleigh conversion of photons, and, therefore, has no direct bearing on the content of this article. If anything, the results of reference [5] of this article - which are dismissed in [20] - can be easily interpreted by means of the quantum Rayleigh conversion of photons as described in Section 2 above and the Appendix B below.

Another example of independent qubits replacing the annihilated states of entangled photons is outlined in the Appendix A below for the case of two-photon quantum interference [21].

\section{Conclusions}

Quantum Rayleigh conversions of photons in dielectric media provide a physically meaningful explanation for experimental results of statistical and "nonlocal" quantum correlations supposedly associated with entangled states of photons. Single and independent qubits replace the annihilated entangled states and provide identical correlation functions between two sets of polarization-related measurements carried out far apart from each other. This physically meaningful analysis raises significant doubts about the existence of photonics-based quantum nonlocality processes.

\section{Appendix A-Coincident counts of two-photon states with single and independent qubits for fourth-order quantum interference}

As an additional application of independent and single qubits generated through quantum Rayleigh conversion of photons we consider the case of two-photon quantum interference of coincident counts [21]. Two pairs of signal $(s)$ and idler ( $i$ ) waves are emitted by two sources labelled with $k=1$ or 2 . Two photodetectors count, separatrely, the signal photons and idler photons. The joint probability $\mathrm{P}_{12}$ of detecting a signal photon and an idler photon with both detectors in coincidence is [20]:

$$
\mathrm{P}_{12}=\left\langle\Phi\left|E_{s}^{(-)} E_{i}^{(-)} E_{i}^{(+)} E_{s}^{(+)}\right| \Phi\right\rangle
$$

where the field operators are expressed as a superposition of the photon annihilation and creation operators, $\hat{a}$ and $\hat{a}^{\dagger}$ respectively, of the two waves, $j=s$ or $i$, in the following equalities:

$$
\hat{E}_{j}^{(+)}=\hat{a}_{j 1} e^{i \theta_{j 1}}+\hat{a}_{j 2} e^{i \theta_{j 2}}
$$




$$
\hat{E}_{j}^{(-)}=\hat{a}_{j 1}^{\dagger} e^{-i \theta_{j 1}}+\hat{a}_{j 2}^{\dagger} e^{-i \theta_{j 2}}
$$

The composite wave function $|\Phi\rangle$ involved in the evaluation of the probability in Eq. (A1) is the direct product of the four states $(k=1$ or 2$)$;

$$
|\Phi\rangle=\prod_{j, k}\left|\Psi_{j, k}\right\rangle
$$

with the signal and idler qubits impinging on their respective detectors being the superposition of the vacuum or zero-number state and one-photon number state, taking the normalized form, with real coefficients:

$$
\left|\Psi_{j, k}\right\rangle=c_{0 j k}|0\rangle+c_{1 j k}|1\rangle
$$

As the operators $\hat{a}_{j k}$ and $\hat{a}_{j k}^{\dagger}$ act on states with identical indices, the cross terms become:

$$
\begin{aligned}
& \left\langle\Psi_{j, k}\left|\hat{a}_{j k}\right| \Psi_{j, k}\right\rangle=c_{1 j k} c_{0 j k} \\
& \left\langle\Psi_{j, k}\left|\hat{a}_{j k}^{\dagger}\right| \Psi_{j, k}\right\rangle=c_{0 j k} c_{1 j k}
\end{aligned}
$$

After inserting equations (A2) - (A5) into (A1), one finds that an interference term can be identified for $\mathrm{P}_{12}$ as a function of phase shift differences acquired by the photons as they propagate from the sources to the detectors, that is:

$$
\mathrm{P}_{12}(\Theta)=2\left(\prod_{j, k} c_{0 j k} c_{1 j k}\right) \cos \Theta
$$

where $\Theta=\theta_{s 2}-\theta_{s 1}+\theta_{i 2}-\theta_{i 1}$ corresponds to the sum of the phase differences between the signal states and between idler states. Eq. (A6) is functionally similar to the result of [20] (Eq.8) for two-photon quantum interference.

Nonetheless, modulation of the optical path or refractive index may induce loss of photons and could contribute to quantum interference of coincident photon counts by changing one or more of the probability amplitudes in Eq. (A6). For instance, the angle for total reflection in a waveguide my change allowing more photons to escape, or the reflection from a mirror may shift laterally the direction of propagation causing photons to miss the photodetector.

\section{Appendix B - The equations of motion for the quantum Rayleigh conversion of photons}

In terms of the pump number of photons $N_{2}$ and corresponding phase $\varphi_{2}$ the following equations of motion provides the rates of change of the spontaneously emitted photons $N_{1}$ and related phase $\varphi_{1}$ as follows [9-11]:

$$
\begin{aligned}
& \frac{\partial}{\partial t} N_{1}=g_{1} N_{1} \\
& g_{1}=-2 \kappa r_{21} \sin \theta_{21}
\end{aligned}
$$




$$
\begin{aligned}
& \frac{\partial}{\partial t} \varphi_{1}=\kappa r_{21} \cos \theta_{21} \\
& r_{21}(t)=\left(N_{2} / N_{1}\right)^{1 / 2} \\
& \kappa=\frac{k_{o}}{2 n} \iint d x d y \chi^{(1)} f_{1} f_{2} \boldsymbol{e}_{1} \cdot \boldsymbol{e}_{2}
\end{aligned}
$$

where the gain coefficient $g$ includes an overall coupling coefficient $\kappa$ defined in [11] which depends on the polarization states of the photons $\boldsymbol{e}_{1}$ and $\boldsymbol{e}_{2}$. The phase difference between the two waves is $\theta_{21}=\left(\beta_{2}-\beta_{1}\right) z+\varphi_{2}-\varphi_{1}, \beta$ being the propagation constant and $z / t=\mathrm{v}_{\mathrm{p}}$ is the phase velocity. In Eq. (B5), $k_{\mathrm{o}}$ and $n$ specify the free-space wavevector and the effective refractive index, respetivly. It should be noted that Eqs. (B1-B5) describe the physically meaningful process of quantum Rayleigh conversion of photons [9 - 11]. The coupling coefficient of Eq. (B5) indicates that the entire local value of the optically linear susceptibility $\chi^{(1)}$ is involved in the coupling process in the dielectric medium at any point where the two spatial distributions $f_{1}$ and $f_{2}$ overlap, each having units of $\mathrm{m}^{-1 / 2}$, and the squares $f^{2}$ are normalized to a dimensionless unit over the crosssection area. This is in contrast to the physically impossible coupling between two optical waveguides apparently induced by a perturbation of the dielectric constant in the cladding - see reference [11] for details.

\section{References:}

1. Aspect, A., Closing the Door on Einstein and Bohr's Quantum Debate, Physics, 2015, 8, 123-126.

2. Hensen, B.; Bernien H.; Dréau, A. E; Reiserer, A; Kalb, N; Blok, M. S.; Ruitenberg, J; Vermeulen, R. F. L; Schouten, R. N.; Abellán, C.; Amaya, W.; Pruneri, V.; Mitchell, M. W.; Markham, M.; Twitchen, D. J.; Elkouss, D.; Wehner, S.; Taminiau, T. H.; and Hanson, R.; Loophole-free Bell Inequality Violation Using Electron Spins Separated by 1.3 Kilometres, Nature 2015 526, 682-686.

3. Giustina, M.; Versteegh, M.A. M.; Wengerowsky, S,; Handsteiner,J.; Hochrainer, A.; Phelan, K.; Steinlechner, F.; Kofler, J.; Larsson, J.-̊̊.; Abellán, C.; Amaya, W.; Pruneri, V.; Mitchell, M. W.; Beyer, J.; Gerrits, T.; Lita, A. E.; Shalm, L. K.; Nam, S. W.; Scheidl, T.; Ursin, R.; Wittmann, B, and Zeilinger, A.; Significant-Loophole-Free Test of Bell's Theorem with Entangled Photons, Phys. Rev. Lett. 2015, 115, 250401.

4. Shalm, L. K.; Meyer-Scott, E.; Christensen, B. G.; Bierhorst, P.; Wayne, M. A.; Stevens, M. J.; Gerrits, T.; Glancy, S.; Hamel, D. R.; Allman,M. S.; Coakley, K. J.; Dyer, S. D.; Hodge, C.; Lita, A. E.; Verma, V. B.; Lambrocco, C.; Tortorici, E.; Migdall, A. L.; Zhang, Y.; Kumor, D. R.; Farr, W. H.; Marsili, F.; Shaw, M. D.; Stern, J. A.; Abellán, C.; Amaya, W.; Pruneri, V.; Jennewein, T.; Mitchell, M. W.; Kwiat, P. G.; Bienfang, J. C.; Mirin, R.P.; Knill, E. and Nam, S. W.; Strong Loophole-Free Test of Local Realism, Phys. Rev. Lett. 2015, 115, 250402.

5. Qian, X. F.; Little, B.; Howell, J. C.; and Eberly, J. H., Shifting the quantum-classical boundary: theory and experiment for statistically classical optical fields, Optica 2015, 2, 611-615.

6. Griffiths, D. J.; 2005, Introduction to Quantum Mechanics, Publisher: Pearson Prentice Hall.

7. Louisell, W. H.; 1973, Quantum Statistical Properties of Radiation, John Wiley \& Sons.

8. Marcuse, D., 1980, Principles of Quantum Electronics, Academic Press, 1980. 
9. Vatarescu, A., Photonic coupling between quadrature states of light in a homogeneous and optically linear dielectric medium, J. Opt. Soc. Am. B, 2014 ,31, 1741-1745.

10. Vatarescu, A., Phase-Sensitive Amplification with Low Pump Power for Integrated Photonics, OSA Advanced Photonics Congress, 2016, paper ID: IM3A.6.

11. Vatarescu, A., Photonic Quantum Noise Reduction with Low-Pump Parametric Amplifiers for Photonic Integrated Circuits Photonics 2016, 3, 61.

12. Wang, Z.; Wu, H.; Hu, X.; Zhao, N.; Qi Mo and \& G. Li, Rayleigh scattering in few-mode optical fibers, Sci. Rep. 2016, 6, 35844; doi:10.1038/srep35844.

13. Piacentini, F.; Alessio Avella, A.; Rebufello, E.; Lussana, R.; Villa, F.; Tosi, A; Gramegna, M.; Brida, G.; Cohen, E.; Vaidman, L.;5, Degiovanni, I. P. and Genovese, M., Determining the quantum expectation value by measuring a single photon, Nat. Phys., 2017,13, 1191-1194.

14. F. J. Tipler, Quantum nonlocality does not exist, PNAS, 2014, 111 (31), 11281-11286, August 5, 2014; https://doi.org/10.1073/pnas.1324238111.

15. Glauber, R. J. and Lewenstein, M., Quantum optics of dielectric media, Phys. Rev. A, 1991, 43, 467491.

16. Brunner, N.; Cavalcanti, D.; Pironio, S.; Scarani, V. and S. Wehner, S., Bell nonlocality, Rev. Mod. Phys. 2014, 86, 419-478.

17. Garrison, J. C. and Chiao, R. Y., 2008, Quantum Optics, Oxford University Press.

18. Ringbauer, M.; Giarmatzi, C.; Chaves, R.; Costa, F.; White, A. G. and Fedrizzi, A., Experimental test of nonlocal causality, Science Advances 2016, 2, no. 8, 10 Aug 2016; DOI: 10.1126/sciadv.1600162.

19. Ursin, R.; Tiefenbacher, F.; Schmitt-Manderbach, T.; Weier, H.; Scheidl, T.; Lindenthal, M.; Blauensteiner, B.; Jennewein, T; Perdigues, J.; Trojek, P; Ömer, B.; Fürst, M.; Meyenburg, M.; Rarity, J.; Sodnik, Z.; Barbieri, C.; Weinfurter, H. and Zeilinger, A. , Entanglement-based quantum communication over 144 km, Nat. Phys. 2007, 3, 481-486.

20. E. Karimi and R. W. Boyd "Classical entanglement? "Science Magazine, 4 DECEMBER 2015, VOL 350, ISSUE 6265, pp. 1172-1173.

21. Mandel, L., Quantum effects in one-photon and two-photon interference, Rev. Mod. Phys., 1999, 71, S274-S282. 